\title{
Un pionnier de la bibliothéconomie au Canada français Auguste-Marie Morisset, OMI
}

Auguste-Marie Morisset, OMI: A Pioneer of Librarianship in French Canada

\section{Un pionero de la biblioteconomía en el Canadá francés: Auguste-Marie Morisset, OMI}

\section{Richard Greene et Jean LeBlanc}

Volume 46, numéro 3, juillet-septembre 2000

URI : https://id.erudit.org/iderudit/1032655ar

DOI : https://doi.org/10.7202/1032655ar

Aller au sommaire du numéro

\section{Éditeur(s)}

Association pour l'avancement des sciences et des techniques de la documentation (ASTED)

\section{ISSN}

0315-2340 (imprimé)

2291-8949 (numérique)

\section{Découvrir la revue}

\section{Citer cet article}

Greene, R. \& LeBlanc, J. (2000). Un pionnier de la bibliothéconomie au Canada français Auguste-Marie Morisset, OMI. Documentation et bibliothèques, 46(3), 135-142. https://doi.org/10.7202/1032655ar
Résumé de l'article

Cette esquisse biographique célèbre la mémoire du père Auguste-Marie Morisset, OMI, bibliothécaire en chef de l'Université d'Ottawa durant quelque 25 ans. L'Université lui a rendu hommage en donnant son nom au pavillon qui loge sa bibliothèque des arts et des sciences. On ne trouvera ici ni réquisitoire ni plaidoyer, mais un portrait du père Morisset tel qu'il apparaît à travers les sources et les témoignages, avec ses qualités et ses défauts, ses ombres et ses lumières, ses réalisations et ses échecs. Les auteurs ont voulu suivre toutes les étapes de sa vie, mais en insistant particulièrement sur sa carrière dans le milieu des bibliothèques, comme professeur, directeur de bibliothèque, fondateur d'une école de bibliothéconomie, conférencier et homme d'associations.
Tous droits réservés (C) Association pour l'avancement des sciences et des techniques de la documentation (ASTED), 2000
Ce document est protégé par la loi sur le droit d'auteur. L'utilisation des services d'Érudit (y compris la reproduction) est assujettie à sa politique d'utilisation que vous pouvez consulter en ligne.

https://apropos.erudit.org/fr/usagers/politique-dutilisation/ 


\title{
Un pionnier de la bibliothéconomie au Canada français: Auguste-Marie Morisset, OMI
}

\author{
Richard Greene \\ Bibliothécaire en chef \\ Université d'Ottawa \\ Jean LeBlanc \\ Ex-directeur, Bibliothèque Morisset \\ Université d'Ottawa
}

Cette esquisse biographique célèbre la mémoire du père Auguste-Marie Morisset, OMI, bibliothécaire en chef de l'Université d'Ottawa durant quelque 25 ans. L'Université lui a rendu hommage en donnant son nom au pavillon qui loge sa bibliothèque des arts et des sciences. On ne trouvera ici ni réquisitoire ni plaidoyer, mais un portrait du père Morisset tel qu'il apparaît à travers les sources et les témoignages, avec ses qualités et ses défauts, ses ombres et ses lumières, ses réalisations et ses échecs. Les auteurs ont voulu suivre toutes les étapes de sa vie, mais en insistant particulièrement sur sa carrière dans le milieu des bibliothèques, comme professeur, directeur de bibliothèque, fondateur d'une école de bibliothéconomie, conférencier et homme d'associations.

\begin{abstract}
Auguste-Marie Morisset, OMI: A Pioneer of Librarianship in French Canada

This biographical sketch celebrates the life and memory of Father Auguste-Marie Morisset, OMI, chief librarian at the University of Ottawa for over 25 years. The University honoured his memory in naming the building that houses the arts and sciences library in his memory. This article is neither an indictment nor a defence of Father Morisset, but rather a portrait of the man with his strengths and weaknesses, achievements and failures, such as they appear in the source documents and eyewitness accounts. The authors wanted to document his life, placing special emphasis on his career as a librarian, teacher, library administrator, founder of a library school, and member of several professional associations.
\end{abstract}

\begin{abstract}
Un pionero de la biblioteconomía en el Canadá francés: Auguste-Marie Morisset, OMI

Este bosquejo bibliográfico celebra la memoria del padre Auguste-Marie Morisset, OMI, Jefe de bibliotecarios de la Universidad de Ottawa durante unos 25 años. La Universidad le rindió homenaje dando su nombre al pabellón de la biblioteca de Artes y Ciencias. Este trabajo no es acusatorio ni apologético, sino un retrato del padre Morisset tal como aparece en las fuentes y los testimonios, con sus cualidades y sus defectos, sus luces y sus sombras, sus logros y sus fracasos. Los autores han querido seguir todas las etapas de su vida, pero insistir particularmente en la carrera dentro del medio bibliotecario, como profesor, director de biblioteca, fundador de una escuela de biblioteconomía, conferencista y miembro de asociaciones profesionales.
\end{abstract}

Celui qui fut simplement connu par collègues et étudiants comme le père Morisset naquit en 1900 à Fall River, Massachusetts; aujourd'hui, le pavillon ainsi que la bibliothèque des arts et des sciences de l'Université d'Ottawa portent son nom. Son père, Eugène, avait épousé en 1897 une jeune fille de cette localité. Le couple eut 14 enfants et une des filles fut la mère du père Roger Guindon, OMI, recteur de l'Université d'Ottawa de 1964 à 1984. C'est sans doute pour des raisons économiques, comme ce fut le cas pour beaucoup de leurs compatriotes à l'époque, que les Morisset émigrèrent aux États-Unis. Et c'est encore pour améliorer leur sort qu'ils revinrent s'installer au
Témiscamingue, présenté à la fin du $\mathrm{XIXe}$ siècle par des hérauts de la colonisation, tel le curé Labelle, comme une terre d'avenir pour «ceux qui sont des cultivateurs originaires du Canada, ont vécu au pays et pleurent la patrie absente» (Lévesque 1979, 136-149), ce qui correspond bien, semble-t-il, au profil des Morisset.

\section{Le religieux}

Le jeune Auguste fit ses études primaires à Lorrainville, puis commença ses études secondaires à Ville-Marie. Après avoir passé une année au juvénat des Maristes à Iberville pour ensuite fréquenter leur postulat de Saint-Hyacinthe, il entreprit en 1915 ses études classiques au juniorat des Oblats à Ottawa. II prit en 1921 I'habit des Oblats de Marie-Immaculée (OMI) au noviciat de Ville LaSalle. On le retrouve en 1922 au scolasticat d'Ottawa; il y prononce ses premiers vœux, y fait sa philosophie et sa théologie, puis franchit les étapes qui le conduiront au sacerdoce. Ses supérieurs décèlent déjà chez lui (Brouillet 1990, 1-2) les qualités qui le caractériseront: piété, zèle, régularité, facilité d'établir des relations, mais aussi ses faiblesses de tempérament: impressionnabilité, promptitude à condamner, nervosité. Particulièrement perspicace, le père J.M.-R.Villeneuve (le futur cardinal, supé- 
rieur du scolasticat de 1920 à 1930) dira qu'il ne possède pas un "esprit des plus brillants». On trouve donc déjà, à cette époque, l'essentiel de sa personnalité: communicateur mais influençable, travailleur mais parfois superficiel, entreprenant tout en ne mesurant pas toujours, et ses forces et les obstacles à vaincre. II entretient aussi une volumineuse correspondance, donne des conférences, fait preuve de grande curiosité intellectuelle, traits qui ne se démentiront pas non plus par la suite.

Le père Morisset prononce ses vœux perpétuels à Ottawa en 1925. C'est alors qu'il ajoute à Auguste le nom de Marie. Ordonné prêtre en 1927, il est nommé en 1928, après une période de ministère paroissial, vicaire-économe à Saint-PierreApôtre de Montréal. Les années 19281934 seront pour lui une période d'exploration et de recherche de son orientation. Tour à tour ou en même temps vicaire, professeur, aumônier de troupes scoutes, animateur; il semble que dans chacune de ces activités, il prenne conscience de son manque de préparation, de ses limites, qu'il s'agisse de prêcher ou d'enseigner, d'où cette soif de savoir qui le poussera à reprendre ses études (Brillant 1977, 540). De retour à Ottawa en 1929 comme professeur de latin et de français au juniorat, il ne sait pas encore qu'il se fixe pour plus de 40 ans et qu'il ne quittera plus l'orbite universitaire jusqu'à sa retraite en 1971.

II devient l'année suivante, et pour quatre ans, vicaire à la paroisse du SacréCœur. Malgré quelques problèmes de santé, le père commence, dès 1929, à temps partiel, des études en droit canon et en littérature française. L'École supérieure de droit canonique venait d'être créée. Le père Morisset y obtiendra sa licence dès 1930. II poursuivra, par ailleurs, ses études en littérature française jusqu'en 1936, sans jamais obtenir de diplôme. Encore ici se manifeste cet autre trait de son caractère, la volonté de tout faire en même temps, de poursuivre le combat sur tous les fronts. Quoi qu'il en soit, la fréquentation plus suivie de la bibliothèque et de ses collections lui fait réaliser au cours de ces années l'importance de l'organisation, celle de la documentation étant le préalable à celle de la connaissance.

\section{Le bibliothécaire de l'université}

À cette époque, dans les communautés religieuses, les obédiences étaient autant le fruit du hasard ou des besoins que des intérêts ou des capacités du sujet. On peut estimer providentielle la décision prise par le recteur de l'Université de nommer, en septembre 1934, le père Morisset, bibliothécaire en chef de l'Université, poste qu'il conservera jusqu'en 1958. II faut, pour bien évaluer l'ampleur de la tâche, esquisser le contexte des bibliothèques canadiennes durant ces années de crise.

Guy Sylvestre a rappelé, dans une allocution prononcée le 23 octobre 1971, à l'occasion de la grande Fête de l'amitié honorant le père Morisset, que ce dernier était entré dans la profession un an après la publication du rapport Ridington, qui déplorait en 1933 la grande misère des bibliothèques canadiennes. Cette Commission d'enquête, formée par l'American Library Association (ce qui est déjà assez révélateur!) et financée par la Carnegie Foundation (ce qui l'est encore plus!), étudie la situation de tous les types de bibliothèques, province par province. Sept bibliothèques universitaires (dont Ottawa) ne se donnent même pas la peine de répondre au questionnaire. On évalue cependant la collection de l'Université à quelque 30000 volumes (Ridington 1933, 126-130). Par ailleurs, il n'y avait alors qu'une seule école de bibliothéconomie (McGill, fondée en 1904, mais ne décernant le BLS que depuis 1931) assurant la formation professionnelle et aucune association professionnelle nationale (la CLA ne fut fondée qu'en 1946 et l'ACBLF en 1948) permettant aux bibliothécaires de partager leurs préoccupations ${ }^{1}$.

Pauvreté des collections, étroitesse des locaux, budgets minimaux, personnel improvisé: tel était le lot de la plupart des bibliothèques canadiennes, de tous les types et à tous les niveaux. Responsable d'une bibliothèque constituée d'à peine deux salles, le père Morisset prend immédiatement la mesure du défi et décide, en premier lieu, d'apprendre les rudiments de la profession en passant deux jours par semaine à la bibliothèque municipale Carnegie. C'est dans cet environnement qu'il réalise avec encore plus d'acuité qu'on ne peut bâtir une bibliothèque universitaire sans un personnel bien formé.

II montera donc le premier à la ligne de feu. Surmontant ses problèmes de santé, il décide d'abord de consentir les efforts nécessaires pour compléter son baccalauréat, condition indispensable à son admission dans une école de bibliothéconomie. II l'obtiendra en 1935 de l'Université d'Ottawa. Puis il s'inscrit à la session d'été du Department of Library Science de l'Université du Michigan à Ann Arbor. Comme il le dira lui-même en 1952: «il me fallait approfondir l'A.B.C. d'une science et d'une technique qui ne s'improvisent guère [...] ". II était devenu évident que l'apprentissage sur le tas ne correspondait plus à l'évolution des choses. Cette conviction, semble-t-il, n'était pas complètement partagée par ses supérieurs, puisqu'on lui refuse, en mai 1936, la permission de participer à un congrès à Richmond, Virginie, en lui soulignant le « danger de faire croire que nous sommes quelque chose en fait de bibliothèque [...] ce qu'il nous faut actuellement, c'est nous organiser, au moins pour ce que nous possédons. Apprendre par la pratique dans des bibliothèques qui ressemblent à la nôtre les notions essentielles".

Les années 1936-1939 seront celles des études en bibliothéconomie poursuivies maintenant à la Columbia University School of Library Service, sans doute parce qu'il s'est avéré plus facile de se déplacer vers New-York que vers Ann Arbor. Ce sont des années difficiles. Le père doit faire l'apprentissage de l'anglais; il se sent isolé dans un milieu complètement étranger, loin de sa famille religieuse et naturelle, et il s'inquiète du sort réservé à sa bibliothèque confiée à un directeur intérimaire.

Les notes des cours de l'été 1936 ne sont pas très bonnes. II obtient de retourner à Columbia pour une année scolaire complète, et Juliette Chabot lui écrit, en septembre 1937: «Je suis mille fois heureuse que vous ayez obtenu une année

1. Fondée en juin 1946, la CLA (Canadian Library Association) fut initialement bilingue, le père Morisset ayant fortement soutenu cette résolution lors du congrès de fondation. Ce n'est qu'en 1958 qu'elle abandonna l'appellation française (Association canadienne des bibliothèques - ACB). Au Québec, la QLA-ABQ (Quebec Library Association-Association des bibliothèques du Québec) fut fondée en 1932, et l'ACBLF (Association canadienne des bibliothèques de langue française) en 1948. 
d'étude à l'Université de Columbia. Vous deviendrez sans doute le plus fort technicien et le plus cultivé de nos bibliothécaires canadiens-français [...]». C'est en mars 1938, dans le cadre de l'un de ses cours, qu'il dépose un travail intitulé Plans for a library for the University of Ottawa, dans lequel s'exprime son optimisme naturel, mais surtout une vision de l'avenir : il y établit un plan de bibliothèque centrale capable de servir 2000 lecteurs, prévoit un accroissement de la collection actuelle (80 000 volumes) $)^{2}$ de quelque 2000 ouvrages par année, dans le but d'en arriver à 140000 volumes dans 30 ans (Brouillet $1990,3)$. En réalité, ce dernier objectif sera atteint en deux fois moins de temps.

Le père Morisset obtient son Bachelor of Science (Library Service) en juin 1938 et complète durant les mois d'été sa scolarité de maîtrise. Reste la thèse, demeurée de longues années en gestation. Un séjour à New York à l'automne 1944 lui permet d'en esquisser enfin une ébauche. Elle porte sur les principes et usages de la rédaction des catalogues, avec étude comparative des catalogues de la Bibliothèque nationale de Paris, du British Museum, de la Library of Congress et du Deutscher Gesamkatalog. Cette première version est terminée en juin 1945. Ce n'est cependant qu'en février 1948 que le père obtiendra son M.S. (Library Service) de l'Université Columbia, après 10 ans de labeur, constamment interrompu par ses autres occupations, sa mission pastorale et ses responsabilités d'enseignant et d'administrateur. On aime à rappeler qu'il fut le premier Canadien français détenteur d'un MLS.

Les mois qui suivirent son retour à Ottawa témoignent d'une intense activité. Le père avait déjà son programme en tête: développement planifié des collections, aménagement fonctionnel des locaux, création d'un catalogue selon les normes, formation du personnel. II met sur pied, dès la fin de 1938, un service de catalogage qui traitera les documents en conformité avec les normes de l'ALA (American Library Association), puis il est chargé de créer un catalogue bilingue. S'ajouteront au fil des années divers services spécialisés: réserve, prêt entre bibliothèques, reliure, audiovisuel, micrographie. Enfin, par la fondation de l'École de bibliothécaires en 1938, qu'il dirigera jusqu'en 1971, il jette les bases d'un établissement de nature à donner un caractère universitaire à une profession jusque-là surtout teintée d'amateurisme. C'est dans le même esprit qu'il avait entrepris, dès 1937, une collaboration avec l'École de bibliothécaires de l'Université de Montréal, où il sera professeur invité et chargé de cours jusqu'en 1962.

Guy Sylvestre révélait, lors de son allocution d'octobre 1971 évoquée plus haut, qu'un rapport confidentiel préparé en 1941 pour une fondation américaine (sans doute la Carnegie Foundation) qualifiait le père de «young, enthusiastic and industrious librarian [...] ardent convert to modern practice». Le père Morisset fera preuve, jusqu'en 1958, année de sa démission du poste de bibliothécaire en chef, d'une activité débordante, d'une énergie sans failles, d'un dynamisme communicatif, et ceci, malgré la pauvreté relative des moyens mis à sa disposition.

Dès la fin des années de guerre, des progrès considérables auront été accomplis. La fondation en 1945 de la Société des Amis de la bibliothèque, recrutés chez les Anciens, assurait un support externe. À l'interne, le père avait réussi à porter à cinq personnes, le personnel, en plus des huit aides-bibliothécaires. Et dans son article publié dans Le Devoir du 8 septembre 1945 , il répète son souhait de construire une nouvelle bibliothèque et il fait état de 135000 volumes, des 450 périodiques et des 1700 usagers enregistrés (Morisset 1945, 4-7).

Le père Morisset multiplie ses activités: participation aux congrès durant les vacances (Bibliographical Society of Canada, ALA, CLA, etc.), conférences à des clubs sociaux, allocutions lors d'inauguration de bibliothèques ou de réceptions pour présentation d'ouvrages, causeries à la radio, responsabilités au sein d'associations ou d'organismes divers, preuve que sa réputation professionnelle se répand à l'échelle du pays. L'année 1947 voit la création de la bibliothèque de médecine (une École de médecine avait été fondée en 1945). Les collections connaissent une croissance satisfaisante: 140000 volumes en 1947, 206000 en 1956.

Cette expansion n'est évidemment pas sans conséquences, et le père revient en 1950 sur la nécessité de créer une bibliothèque centrale, les collections étant maintenant dispersées dans quelque 10 locaux. Les bibliothèques doivent suivre le développement de l'Université et cette dernière voit, de 1953 à 1957, la création de trois autres facultés (Droit civil, Sciences sociales, Common Law). Lourdeur des tâches administratives, poids de l'enseignement aux Écoles d'Ottawa et de Montréal: le père ressent la nécessité de se ressourcer. En 1955, il quitte Ottawa pour un voyage de quatre mois en Europe, dont le but est principalement de participer au Congrès international des bibliothèques et des centres de documentation à Bruxelles du 11 au 18 septembre ainsi qu'à deux autres congrès spécialisés (bibliothèques médicales et musicales) qui s'y tiennent simultanément. Délégué du Canada à titre de membre du Comité consultatif de la Bibliothèque nationale du Canada, il y représente aussi l'ACB/CLA et l'ACBLF. II y fera une communication sur les bibliothèques du Canada. De plus, à titre de boursier du gouvernement fédéral, il visite de nombreuses bibliothèques européennes; il en revient avec l'impression que les bibliothèques européennes sont plus riches que les nôtres, mais moins avancées techniquement que celles de nos voisins américains.

II supervise en 1956 le déménagement de la bibliothèque. Même s'il ne s'agit pas encore de la réalisation de son vœu, c'est-à-dire de voir la construction d'une bibliothèque centrale pour regrouper la plupart des collections, ce déménagement constitue un progrès certain. Mais la tâche est devenue trop lourde (Brouillet $1990,3-4)$. Ses supérieurs, tout en ne niant pas ses réalisations, jugent que ses énergies sont trop éparpillées, qu'avec la direction de l'École conjuguée à celle de la bibliothèque, le professeur en porte trop sur ses épaules. La planification laisse à désirer et il ne réussit plus à répondre aux besoins grandissants, tant en locaux et en collections qu'en personnel. La gestion interne suscite de plus en plus de mécontentement autant chez le corps professoral que chez les élèves: les livres sont catalogués avec trop de retard, les fichiers incomplets. Le père n'a pu gérer la croissance avec suffisamment d'efficacité, et on ne peut s'empêcher de voir dans cette situation le prélude des événements des

2 Ces statistiques ne doivent pas faire illusion. L'Université s'identifiant avec les Oblats, le décompte des collections inclut aussi souvent celles du séminaire universitaire, du scolasticat et du juniorat. Ainsi les chiffres donnés plus loin dans le texte pour 1947 se répartissent vraisemblablement comme suit: 140000 volumes au total, soit 60000 pour l'université, 30000 pour le séminaire universitaire, 40000 pour le scolasticat et 10000 pour le juniorat. 
années 1966-1970 à l'École de bibliothécaires, alors qu'on lui reprochera aussi un manque de rigueur administrative. On décide de le remplacer à la tête des bibliothèques, qui comprennent alors plus de 200000 volumes et qui sont dotées d'un budget annuel d'acquisitions de $80000 \$$. Son successeur, le père Drouin, OMI, reçoit pour mandat de planifier la centralisation éventuelle des bibliothèques dans un seul édifice, projet qui ne sera réalisé (partiellement) qu'en 1972. Le père Morisset devient, au début de 1959, président du nouveau Conseil général des bibliothèques. Poste purement honorifique. On a voulu, en réalité, lui signifier qu'il devait concentrer ses énergies sur l'administration de l'École.

\section{L'École de bibliothécaires}

«En 1938, le père Auguste Morisset, bibliothécaire de l'université, reconnaît le besoin de formation permanente pour les nombreux bibliothécaires de la capitale nationale. Il obtient que la faculté des Arts offre des cours à temps partiel en vue du baccalauréat en bibliothéconomie [...]» (Guindon 1995, 20). Ainsi sont résumés succinctement les deux volets initiaux du projet. II s'agira d'un programme principalement axé sur les besoins des praticiens de la région, et parce qu'il s'agit de personnes ayant un emploi, les cours seront à temps partiel.

Une enquête menée en 1967 sur les caractéristiques des diplômés de l'École depuis ses débuts démontre qu'on avait visé juste (Brillant 1977, 561). Le groupe est surtout constitué d'hommes, originaires principalement de l'Ontario et du Québec, plutôt mûrs, mi-trentaine, bilingues et mêmes polyglottes, qui font leurs études à temps partiel. II y a un fort contingent d'immigrants (quelque $30 \%$ de naturalisés). Un tiers détenait déjà un diplôme d'études supérieures. Ces étudiants travaillent majoritairement dans trois types de bibliothèques: universitaires ou collégiales, gouvernementales ou spécialisées.

On peut en conclure que le contexte se prêtait à l'initiative. Les bibliothèques gouvernementales, après la guerre, commençaient à proliférer. II y avait une forte demande de recyclage (Henderson 1977, 406-407). Le programme élaboré par le père Morisset s'inspirait des programmes de McGill et de Toronto. L'École de McGill, fondée en 1904, ne donnait à l'origine que des cours de quelques semaines. Elle ne décerne le Bachelor in Library Science qu'en 1931, une fois l'agrément obtenu l'année précédente: Les premiers BLS de Toronto, qui a obtenu l'agrément en 1937, reçoivent leur diplôme cette même année. Montréal est encore trop jeune pour servir de modèle: son École de bibliothécaires ne fut fondée qu'en 1937, mais n'eut aucun directeur ou professeur à temps plein jusqu'en 1961, où elle devint École de bibliothéconomie (Denis 1968, 152).

Le père a probablement en tête de créer une école bilingue (Brouillet 1990,4) et, en principe, le corps professoral autant que les étudiants devaient l'être, au moins passivement (parler une langue et comprendre l'autre), ce qui ne veut pas dire que le programme lui-même le reflétait. La plupart des cours réguliers seront donnés, au moins jusqu'en 1971, en anglais seulement. Elle ne sera donc malheureusement, dans les faits, qu'unilingue anglaise. Les cours se donnaient le soir et s'adressaient à deux catégories d'étudiants: les étudiants sans diplôme universitaire préalable pouvaient obtenir un certificat et ceux en possession d'un diplôme de premier cycle pouvaient poursuivre le BLS. Le fait que les cours ne soient donnés qu'à temps partiel explique qu'aucun diplôme n'ait été décerné avant 1942, car ce régime exige quatre ans de scolarité. II ne pouvait en être autrement, puisque le père assurait le gros de l'enseignement tout en exerçant ses fonctions de directeur de l'École et de bibliothécaire de l'Université.

On peut distinguer trois périodes dans l'histoire de l'École (Brillant 1977, 539-576). Les débuts, de 1938 à 1950, furent, par la force des choses, modestes. Directeur, professeurs et étudiants œuvrent à temps partiel. Le père, qui donne la plupart des cours, doit les préparer en vitesse (Brouillet 1990, 4); cependant, il a l'intelligence de s'entourer au départ d'une bonne équipe, puisque figurent parmi les premiers professeurs invités le bibliothécaire parlementaire, Félix Desrochers, l'archiviste national, Gustave Lanctôt ainsi que deux bibliothécaires de la Municipale de Montréal, Juliette Chabot, qui deviendra une grande amie, et MarieClaire Daveluy, cofondatrice l'année précédente de l'École de bibliothécaires de l'Université de Montréal. II entretient ainsi des liens étroits avec d'autres institutions, et des réseaux se créent. Trois cours sont offerts chaque année, deux soirs par semaine. Il y a 12 élèves en 1938-1939, mais on constate une baisse des effectifs durant la guerre: trois diplômes sont décernés en 1942 et trois en 1949. L'École n'aura en propre jusqu'en 1966, ni salles de cours ni bibliothèque. Le premier programme met l'accent, entre autres sur le catalogage et la classification, la référence, la gestion des bibliothèques, le choix des livres et l'histoire du livre.

La période 1950-1959 voit d'importants développements. Le programme offert par la faculté des arts avait été regroupé en 1949 en une École de bibliothéconomie reconnue par le Sénat de l'Université. Un programme de maîtrise à temps partiel est établi dès 1950, le programme de premier cycle à temps plein est offert à partir de 1951, et le premier diplôme sous ce régime est décerné en 1952. Des cours de jour se donnent pour la première fois. Des cours d'été sont offerts dès 1952 et ils deviennent réguliers à partir de 1959. Les quatre journées d'étude tenues ce même été comptent 71 inscriptions. Les cours du soir continuent. Un premier professeur est attaché à l'École en 1953. L'École comptait, en 1959, 11 étudiants à temps complet et 32 à temps partiel. Elle avait jusqu'alors décerné en moyenne deux baccalauréats par année.

Troisième période, l'École prenant de l'expansion doit se doter de structures «plus permanentes », d'autant qu'on pense sérieusement à demander l'agrément. Le père Morisset a beaucoup de projets: embaucher du personnel, réviser le programme, améliorer les locaux, recruter des conseillers du milieu (Brillant 1977, 549). II avait profité de son voyage de 1955 pour faire une analyse comparative des programmes de diverses écoles européennes. Un secrétariat est créé en 1960 . Un autre professeur à temps plein, le Dr Gerhard Richard Lomer, est embauché en 1959. Ancien directeur de l'École de McGill, il remplit aussi le poste de directeur adjoint. À Enid Ringrose, embauchée en 1953, et au $D^{r}$ Lomer se joindront le $D^{r}$ Georges Gerych en 1961 et Anna Marteinsson en 1964.

Le corps professoral ainsi renforcé, on peut se pencher sur la révision du cursus. Le programme de certificat est aboli et remplacé par un cours de trois sessions d'été en français seulement. Ce pro- 
gramme aura produit 93 diplômés en 1968 , provenant surtout des bibliothèques scolaires. De nouveaux cours obligatoires (types de bibliothèques, services techniques, information et bibliothèques) et des cours facultatifs (bibliothèques spécialisées: écoles, hôpitaux, collèges et universités) sont ajoutés. Le nombre de diplômés augmente: 15 en 1960 et 40 en 1965. Le programme de maîtrise, pour sa part, sera modifié en 1964 et en 1968. Enfin, un Comité consultatif est créé, dont feront partie, entre autres, Claude Aubry, conservateur de la Bibliothèque publique Carnegie d'Ottawa et Jack Ernest Brown, le bibliothécaire scientifique national.

Ainsi apparemment rebâtie sur des assises plus solides, l'École se croit en bonne posture pour le processus d'agrément, sollicité officiellement en 1964. C'est en 1966 que les trois visiteurs-enquêteurs du Committee on Accreditation de l'American Library Association se présentent à l'École pour une inspection de trois jours. II s'agit du père Paul-Émile Filion, SJ, bibliothécaire en chef de l'Université Laurentienne et de deux Américains, dont le Dr Andrew D. Osborn, qui préside le Comité. Juillet 1966: coup de tonnerre. Le Comité refuse l'agrément. II demande de renouveler le corps professoral, de remanier le programme d'études, tout en jugeant satisfaisants budget, bibliothèque et gestion générale. Ce ne sont donc pas les infrastructures, mais bien les deux composantes essentielles de l'aspect académique qui sont remises en cause.

Le Comité avait-il, comme on l'a soutenu (Brillant 1977, 553-554), des préjugés attribuables à sa composition même, puisque la réalité canadienne et l'aspect de bilinguisme (même inchoatif) étaient étrangers aux Américains et que le père Filion n'avait aucune expérience de l'enseignement dans une École de bibliothécaires? Ces rumeurs, et d'autres semblables, ont couru durant des années et certains ont avancé les hypothèses les plus farfelues.

La réalité revêt d'autres couleurs et apparaît bien différente. Le Comité a produit un rapport fouillé, bien argumenté, analysant, entre autres, chacun des cours inscrits au programme. II possède toutes les caractéristiques de l'objectivité 3 . II reconnaît d'emblée que «The Ottawa Library School is virtually the single-handed achievement of its Director [...]". Mais il porte sur l'ensemble un jugement relative- ment sévère, que ceux qui ont vécu cette période de l'intérieur ne peuvent qu'entériner : [...] M.A. programme inadequate [...] lack of strong electives [...] teaching methods quite conventional [...] curriculum not well structured [...]". Et il identifie sans ambages le cœur du problème: « [....] greatest single need of the School is the strengthening of the faculty [...] ». En d'autres termes, la relève est loin d'être assurée ni du côté académique ni du côté administratif. La moyenne d'âge du corps professoral frôle les 59 ans. Le directeur a 66 ans et le directeur adjoint 84 !

Loin de fermer complètement les portes, le Comité dit espérer qu'une fois les réformes fondamentales accomplies, le processus d'agrément pourra reprendre dans quelques années. Effectivement, il approuve en 1968 une deuxième demande et prévoit une visite d'inspection au printemps 1969. Mais l'administration de l'Université demande qu'on retarde cette visite pour se donner le temps de nommer un nouveau directeur adjoint et de recruter de nouveaux professeurs. En ce sens, le recteur, le père Guindon, avait raison de penser en 1966 que les membres du Comité avaient imposé trop de conditions difficiles à réaliser immédiatement, et même à moyen terme. Une telle «renaissance » ne pouvait qu'être œuvre de longue haleine.

La vie cependant continue et on ne désespère pas de l'avenir. L'École déménage en 1966 dans ses nouveaux locaux avec sa bibliothèque et son premier bibliothécaire. Cinquante baccalauréats sont décernés en 1967. Une aile nouvelle, destinée à mieux loger la bibliothèque, sera construite en 1969: la bibliothèque s'est enrichie de quelque 1000 volumes par année de 1966 à 1971, et en comptera 13430 en 1972 , ce qui veut dire qu'elle aura doublé sa collection en cinq ans. Les derniers cours d'été sont offerts en 1966 à une centaine d'étudiants, principalement originaires du milieu scolaire. Ce programme passera l'année suivante sous la juridiction de l'Éducation permanente.

On procède en 1967 à un autre remaniement du programme d'études: plusieurs cours sont portés à deux crédits et de nouveaux cours optionnels sont ajoutés (littérature des humanités, sciences, sciences sociales). L'année suivante, on retrouve au programme le premier cours Information storage and retrieval, qui marque l'irruption de l'informatique dans les bibliothèques. L'année 1970 est impor- tante à plusieurs égards: un premier étudiant à temps plein s'inscrit au MLS, alors que le nombre d'élèves inscrits au BLS passe à 104 (ils étaient 12 en 1938). L'année suivante, on compte 91 étudiants à temps complet, 47 à temps partiel; 90 baccalauréats seront décernés cette année-là.

À vrai dire, le père Morisset ne s'est jamais vraiment relevé de la sévérité du coup porté par le Committee on Accreditation et il décide de passer le flambeau. II prendra sa retraite de l'Université le 30 juin 1971, après presque 35 années à la barre de l'École. Peter Havard-Williams, recruté en Grande-Bretagne, est nommé pour lui succéder et il entre officiellement en fonction en juillet. II s'attaque à la préparation d'un nouveau programme, recrute des professeurs, mais pour des raisons encore obscures - peut-être jugeait-il le défi impossible à relever - il quitte l'École à la mi1972. Deux autres professeurs remettent en même temps leur démission. En mai 1972, dans son annonce au Sénat, le recteur, le père Guindon, ne cache pas que «ces démissions placent l'Université dans une situation très délicate et très pénible». Dès lors, on décide ce qui suit: mise en veilleuse du lancement du programme de maîtrise de deux ans en septembre 1972, suspension de toute nouvelle admission au baccalauréat, création d'un comité «pour examiner le futur de l'École de bibliothécaires en rapport avec les besoins du marché de la main-d'œuvre et les écoles existantes dans l'Ontario et le Québec".

Ce comité, présidé par le vice-recteur adjoint (Recherche et Planification), J.-M. Joly, comprend aussi Marcel Hamelin, vice-doyen aux Études supérieures, John Hare, professeur aux Lettres françaises, le père Francis $B$. Wallis, OMI, administrateur de l'École depuis le départ de M. Havard-Williams, de l'extérieur, Daniel Reicher, bibliothécaire en chef de l'Université de Montréal et Guy Sylvestre, le bibliothécaire de la Nationale. Un premier rapport intérimaire est déposé à la réunion de 1972 du Comité exécutif du Sénat et le rapport final est remis en novembre 4.

Contrairement à ce que plusieurs ont cru et affirmé, le Comité ne recommande

3. ALA Committee on Accreditation. Report on the University of Ottawa Library Scbool. April 1966.

4. Comité d'étude sur l'enseignement de la bibliothéconomie à l'Université d'Ottawa. Rapport. Novembre 1972. 
pas une abolition définitive de l'École, mais propose plutôt de fermer l'École actuelle tout en soulignant qu'il serait désirable d'en créer une nouvelle, vraiment bilingue, avec une équipe professorale reconstituée. Le Comité reconnaît, d'une part, que le bilinguisme de l'École n'était que façade, trahissant ainsi le caractère officiel de l'Université et, d'autre part, que le programme existant était loin de répondre aux mêmes exigences académiques que celui des autres écoles ontariennes. En un mot, programme au rabais, ne répondant pas aux normes linguistiques et académiques de l'Université. Le Comité confirmait ainsi, six ans plus tard, les conclusions du rapport du Committee on Accreditation. Mais le défi s'avérait sinon insurmontable, du moins gigantesque, d'autant plus que l'année suivante le Conseil des universités de l'Ontario recommandait de « restrict graduate programs to the universities of Toronto and Western Ontario".

L'approche du Comité était donc logique, mais non nécessairement des plus réalistes dans l'immédiat. Toutes les facultés de l'Université prenaient de l'essor, avec un corps professoral de plus en plus compétent. II aurait fallu restructurer le cursus et consentir à faire des efforts considérables pour recruter des professeurs bilingues et convenablement diplômés, pour tout dire renouveler complètement le corps professoral, puisque plusieurs des professeurs en exercice n'étaient qu'à quelques années de la retraite. II aurait fallu rehausser les exigences imposées aux candidats à l'admission et affronter sur ce terrain la concurrence de Toronto, de Western, de McGill et de Montréal. II aurait fallu reprendre à zéro le processus d'agrément. La réussite d'aucun de ces objectifs n'était garantie. L'École pour tout dire était devenue embarrassante pour l'Université. Suspendu, le programme fut officiellement aboli le 30 juin 1976.

Les autopsies ne sont jamais faciles. Jusqu'à quel point le père Morisset portet-il la responsabilité de l'effondrement de ce qui aura été l'œuvre de toute une vie? Lassitude? Fidélité compréhensible mais néfaste à ce que d'aucuns ont qualifié de clique ou de chapelle? On peut juger que s'il avait réagi avec plus de vigueur et de célérité aux recommandations de 1966, écarté ses plus proches collaborateurs et renouvelé son équipe, les choses n'en seraient pas venues là. Fut-il prisonnier de son paternalisme, victime de son bon cœur, sa nature ne lui permettant pas de brusquer ou de heurter quiconque? On peut le penser. Quoi qu'il en soit, l'héritage compte, et il n'est pas négligeable. L'École aura durant son existence décerné 616 baccalauréats (le premier en 1942) et 12 maîtrises (la première en 1954). Elle a donc doublé ses diplômés en cinq ans, puisqu'elle en comptait 309 en 1967 (Denis 1968, 153). Le père a présidé à la formation de quelques générations de bibliothécaires, dont plusieurs francophones retournés travailler au Québec. Ils ont honoré leur profession et ne l'ont cédé en rien à ceux qui ont obtenu leur diplôme d'écoles agréées.

\section{La retraite}

L'année 1971 fut celle de tous les hommages. Le père Morisset est nommé professeur émérite. Lors de la collation des grades du 26 mai, le recteur, le père Guindon, lui rendit ce témoignage appuyé, synthèse de ce que furent l'homme et l'œuvre:

Son travail infatigable, sa constante recherche de ce qui peut améliover la profession et la discipline, son bumanité à laquelle tous les besoins et toutes les détresses pouvaient faire appel avec l'assurance de ne jamais le trouver insensible, sa bonne bumeur et son bumour ont toujours été appréciés par ceux qui travaillaient avec lui et par ceux qui le voyaient à l' auvre.

Le samedi 23 octobre, dans la salle de bal du Château-Laurier, se tient la grande «Fête de l'amitié et de la reconnaissance" organisée par le Cercle des Anciens de l'École. On y célèbre en même temps le $71^{\circ}$ anniversaire de naissance du père. Le banquet réunit quelque 350 collègues, étudiants et amis, en présence de la chancelière de l'Université, Pauline Vanier, accompagnée de grands noms de la profession comme Guy Sylvestre et Brian Land, doyen de la Faculty of Library and Information Science de l'Université de Toronto.

C'est à cette occasion que le recteur, le père Guindon, annonce que le nouveau pavillon et la nouvelle bibliothèque de l'Université seront nommés en l'honneur du père Morisset. Suit le dévoilement de son portrait que l'on retrouve aujourd'hui dans la salle du Conseil du Réseau de bibliothèques. Dans son allocution, Guy Sylvestre, directeur de la Bibliothèque nationale, situe l'action professionnelle du père Morisset: «He has always worked towards rapprochement-rapprochement between librarians for improved performance, rapprochement between the two majority linguistic groups, as well as rapprochement between Canadian and American libraries".

Le père a des plans de retraite: faire un rapport aux Nations-Unies sur les besoins en documentation des pays en voie de développement et assurer un rapprochement de la formation aux divers volets de la profession (bibliothécaires, spécialistes en informatique, documentalistes, etc.). Ce furent des années d'étude, de petits voyages, de lecture et de ministère, et il continua de s'intéresser de près à la profession. Les honneurs et les célébrations se multiplient. Le père Morisset est nommé, en janvier 1976, l'un des 60 récipiendaires de l'Ordre du Canada par le gouverneur général, Jules Léger; on le retrouve à Rideau Hall pour recevoir sa décoration avec les autres membres de cette promotion qui comprend des personnalités comme le peintre, William Kurelek, l'acteur, Jean Gascon et l'ancien ministre libéral, Paul Martin. II reçoit l'année suivante la médaille du jubilé d'argent de l'accession d'Elizabeth II à la couronne (en. 1967, il avait déjà reçu la médaille du Centenaire de la Confédération). II est élu, le 13 juin 1979, membre honoraire de la Société bibliographique du Canada.

La santé du père avait toujours été fragile. Bourreau de travail, il ne s'est jamais ménagé, preuve que le labeur ne tue pas. Mais, en 1985, son état de santé commence à se détériorer plus sérieusement. II est hospitalisé 5 mois en 1987. Ses dernières années voient croître sa surdité et les troubles cardiaques et pulmonaires se font plus fréquents. Le père Morisset est décédé à l'Hôpital général d'Ottawa le 17 juillet 1989. Son neveu, le père Roger Guindon, a rappelé dans son éloge funèbre une grande constante de l'existence du père Morisset: «Sa vie intense de bibliothécaire n'a pas alourdi ni diminué sa vie sacerdotale et religieuse".

\section{L'implication personnelle}

Homme public, le père Morisset eut aussi une vie privée. Sa participation ac- 
tive à la vie des associations professionnelles, ses publications, sa correspondance, attestent des deux facettes de son existence.

Il fit partie, au cours de sa carrière, d'une vingtaine d'associations nationales et internationales de divers types: bibliothéconomie, bien sûr, mais aussi généalogie, littérature, géographie. Membre très actif au sein de ces associations, il exerce aussi des fonctions administratives au sein de plusieurs de ces groupes: président de la Library Association of Ottawa en 1943-1944, conseiller de l'ALA en 1954-1955, président de l'ACBLF en 19601961, président de la Société bibliographique du Canada en 1950-1951. On fait aussi appel au père pour devenir membre du Comité consultatif national chargé, en 1948, de planifier le Centre bibliographique canadien. En 1951, ce Comité remettra un rapport au Secrétaire d'État, rapport qui conduira à la création de la Bibliothèque nationale du Canada (Donnelly $1973,93)$. Le père fera ensuite partie, de 1952 à 1959, du Conseil consultatif de la Bibliothèque nationale du Canada. II était devenu avec les années un bibliothécaire nationalement et même internationalement connu, comme en font foi ses notices biographiques parus dans divers Who's who de 1940 à 1980 .

II trouvait malgré tout le temps d'écrire. Les archives oblates conservent un grand nombre de notes de lecture ou de méditations rédigées de 1921 à 1943. II s'agissait là d'écrits personnels. II n'a, durant sa vie professionnelle, que peu de loisirs pour s'adonner à la recherche et rédiger de très longs textes. Villeneuve (1964) et Rajan (1965) n'en recensent pas moins quelque 70 publications entre 1934 et 1964, soit en moyenne deux par année, la plupart entre une et trois pages, quelquesunes allant jusqu'à dix. Ce sont des textes appartenant à divers genres, reliés le plus souvent aux questions professionnelles. Le tiers de ses textes se compose de comptes rendus. Le reste comprend des articles de périodiques parus en majorité dans le Bulletin de l'ACBLF, mais aussi dans Ontario Library Review, CLA Bulletin, Catholic Library World. Ils traitent en bonne partie de la vie associative (allocutions, programmes de présidence, activités des associations, rapports d'enquête, résumés de congrès, etc.), quelques-uns de la bibliothèque de l'Université ou de l'École de bibliothécaires.
Seuls une dizaine d'articles, publiés sur une période de 10 ans, entre 1950 et 1960 , abordent des questions de fond (aspects intellectuels du catalogage, culture et devoirs des préposés au prêt et à la référence, mission culturelle de la bibliothèque publique, choix des livres, technique et formation professionnelle, rôle de la bibliothèque de recherche dans la civilisation contemporaine, etc.), élaborant d'ailleurs pour la plupart des thèmes de congrès. On sent par conséquent, ici encore, l'homme pressé: il s'agit davantage de remarques que d'analyses. Plusieurs articles d'encyclopédie témoignent également de l'activité littéraire du père Morisset sur les grandes bibliothèques européennes et des communautés religieuses canadiennes. On retrouve aussi quelques préfaces ou bibliographies annotées. Cet ensemble de textes très inégaux exprime des préoccupations professionnelles sincères, un vif intérêt pour tous les aspects de la vie des bibliothèques, mais révèle souvent, d'autre part, que le temps manquait à l'auteur pour approfondir son sujet.

Tel fut le père Morisset: montrant par l'exemple que stabilité et ténacité peuvent accomplir de grandes choses et donnant la leçon qu'il faut savoir s'entourer et ne pas tenter de tout faire seul. Un homme à multiples facettes, prêtre, religieux, chercheur, administrateur, humaniste, éducateur. Ou encore, comme l'a décrit une collègue: «Nature joviale, enthousiasme à bondissements continus, humour normand, satires à la française” (Legault 1959 , 9). Homme de rapprochement, catalyseur des énergies, agent de liaison, ambassadeur de la profession.

II ne fut pas un gestionnaire hors pair. Très fidèle à son entourage, il se révélait souvent paternaliste, reflétant en cela la mentalité de son époque. Sans formation universitaire très poussée, ce n'était ni un grand intellectuel ni un grand érudit. Certains ont pensé qu'il aurait gagné en profondeur ce qu'il aurait ménagé en dispersion. On pourrait dire de lui, mutatis mutandis, ce qu'on a écrit du père Georges-Henri Lévesque, OP: «Plutôt qu'un chercheur, il était un homme d'action qui menait un double combat: imposer l'influence de sa faculté, contribuer à changer la collectivité environnante » (Dumont 1977, 79). C'est à ce titre qu'il mérite le nom de pionnier de la bibliothéconomie au Canada français.

II fut certainement une grande figure de la profession pendant plusieurs décen- nies. Son nom est celui le plus fréquemment cité, après celui du père Edmond Desrochers, SJ, et de Marie-Claire Daveluy, dans le volume Mélanges offerts en 1977 au père Desrochers (Chartrand $1977,833)$. Sa foi dans le livre comme instrument de transmission du savoir était entière: "C'est dans la bibliothèque que se retrouve l'œuivre humaine du passé. Elle est la glèbe où l'homme d'aujourd'hui plante la récolte de l'homme de demain. Elle relie par delà le temps et l'espace toutes les générations successives de l'humanité, tout ce que chaque culture a laissé de permanent. Elle révèle au chercheur l'évidence de la solidarité humaine».

\section{Sources consultées}

Brillant, Louis-G. 1977. L'École de bibliothéconomie de l'Université d'Ottawa: les grandes lignes de son histoire. In Livre, bibliothèque et culture québécoise; mélanges offerts à Edmond Desrochers, s.j. sous la direction de Georges-A. Chartrand. Montréal: ASTED. vol .II, p. 539-576.

Brouillet, Roger, OMI. 1990. Auguste-M. Morisset, Oblat de Marie Immaculée, 1900-1989. (Notices biographiques). Ottawa: Province Saint-Joseph. 8p. Dactylographié.

Denis, Laurent-G. 1968. La formation des bibliothécaires de langue française du Québec. In: Le bibliothécariat au Canada de 1946 à 1967; hommages à Elizabeth Homer Morton. Ed. par Bruce Peel. Victoria: CLA. p.149-161.

Donnelly, Dolores F. 1973. The National Library of Canada. Ottawa: Canadian Library Association.

Dumont, Fernand. 1997. Récit d'une émigration. Montréal: Boréal.

Filion, Paul-Émile, SJ. 1968. University libraries before and after Williams. In Le bibliothécariat au Canada.... op. cit., p. 73-85.

Guindon, Roger, OMI. 1995. La dualité linguistique à I'Université d'Ottawa. Volume 3: 1936-1965: coexistence féconde. Ottawa: Presses de I'Université d'Ottawa.

$$
\text { 1998. Entrevue accordée le } 25 \text { février. }
$$

Henderson, Mary E. P. 1977. Professional library education in Canada. In Canadian libraries in their changing environment. Ed. by Loraine SpencerGary, Carl Gary. Toronto: York University. p. 394-419.

Legault, Agathe, avec la collaboration de Anna Marteinsson. 1959. Le R.P. Auguste-M. Morisset, OMI: ouvrier du métier, ambassadeur de la profession. Bulletin de l'ACBLF 5(1): 3-5, 9.

Lévesque, Robert et Robert Migner. 1979. Le curé Labelle: le colonisateur, le politicien, la légende. Montréal: La Presse.

Morisset, Auguste-M., OMI. 1945. La bibliothèque de I'Université d'Ottawa. [Montréal: Le Devoir]. Tiré à part d'un article paru dans Le Devoir du 8 septembre 1945.

Oblats de Marie-Immaculée. Archives Deschâtelets, Ottawa. Fonds A.-M. Morisset (HEC 3861.A92C, 
4 boîtes (général) et .A926, 4 boîtes (correspondance).

Rajan, Kamala. 1965. A supplementary list of writings of Reverend Auguste-M. Morisset, O.M.I. Ottawa: University of Ottawa, Library School.

Ridington, John, Chairman. 1933. Libraries in Canada; a study of library conditions and needs. By the Commission of Enquiry [of the American Library Association]. Toronto: Ryerson Press.

Université d'Ottawa. Archives. Fonds École de bibliothécaires. Fonds Sénat.

Villeneuve, Jocelyne. 1964. Le Révérend Père Auguste-M. Morisset: sa contribution dans le $d$ omaine de la bibliothéconomie. Une bibliographie annotée. Ottawa: Université d'Ottawa, École de bibliothécaires. $5 p$.

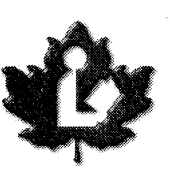

\section{La Passerelle des bibliothèques canadiennes ...connecter les bibliothèques canadiennes} est un centre multiservice de renseignements sur les bibliothèques canadiennes, créé afin de guider les utilisateurs vers l'information dont ils ont besoin et ce, de façon rapide et efficace.

La Passerelle fournit, au moyen du Web, un accès aux catalogues des bibliothèques canadiennes, aux politiques de prêt entre bibliothèques et à la facturation des services des bibliothèques canadiennes, au répertoire des cibles canadiennes Z39.50, au Répertoire des collections spécialisées conservées dans les bibliothèques canadiennes, aux sites Web collectifs des bibliothèques canadiennes et d'autres sites Web d'intérêt. Vous pouvez :

- trouver des renseignements en sciences de l'information et sur les catalogues en ligne dont resAnet, le catalogue en ligne de la Bibliothèque nationale

- effectuer des recherches dans les catalogues collectifs canadiens, y compris Accès AMICUS

- effectuer des recherches dans le Catalogue collectif canadien virtuel (Cccv) par l'entremise de la passerelle Z39.50

- effectuer des recherches au sujet des politiques de prêt entre bibliothèques et de la facturation des services

- effectuer des recherches par sujet à l'aide du Répertoire des collections spécialisées

La Passerelle des bibliothèques canadiennes est le premier lien aux renseignements sur les bibliothèques canadiennes.

Trouvez rapidement des renseignements sur les bibliothèques !

\section{Essayez ce lien maintenant ! Ajoutez-le à vos signets ! http://www.nlc-bnc.ca/gatepasse/}

Pour de plus amples renseignements, veuillez communiquer avec Marketing et édition par téléphone au (613) 995-7969 ou par télécopieur au (613) 991-9871. 